\title{
Structure and regulation of the omega-3 polyunsaturated fatty acid synthase genes from the deep-sea bacterium Photobacterium profundum strain SS9
}

\author{
Eric E. Allen and Douglas H. Bartlett
}

Center for Marine

Biotechnology and

Biomedicine, Marine Biology

Research Division, Scripps

Institution of

Oceanography, University of

California, San Diego, La

Jolla, CA 92093-0202, USA
Author for correspondence: Douglas H. Bartlett. Tel: +1 858534 5233. Fax: +1 8585347313. e-mail:dbartlett@ucsd.edu

Omega-3 polyunsaturated fatty acids (PUFAs) such as eicosapentaenoic acid (20:5n-3; EPA) and docosahexaenoic acid (22:6n-3; DHA) have been shown to be of major importance in the promotion of cardiovascular health, proper human development and the prevention of some cancers. A high proportion of bacterial isolates from low-temperature and high-pressure marine environments produce EPA or DHA. This paper presents the sequence of a 33 kbp locus from the deep-sea bacterium Photobacterium profundum strain SS9 which includes four of the five genes required for EPA biosynthesis. As with other bacterial pfa (polyunsaturated fatty acid) genes, the deduced amino acid sequences encoded by the SS9 genes reveal large multidomain proteins that are likely to catalyse EPA biosynthesis by a novel polyketide synthesis mechanism. RNase protection experiments separated the SS9 pfa genes into two transcriptional units, $p f a A-C$ and $p f a D$. The $p f a A$ transcriptional start site was identified. Cultivation at elevated hydrostatic pressure or reduced temperature did not increase pfa gene expression despite the resulting increase in percentage composition of EPA under these conditions. However, a regulatory mutant was characterized which showed both increased expression of pfaA-D and elevated EPA percentage composition. This result suggests that a regulatory factor exists which coordinates $p f a A-D$ transcription. Additional consideration regarding the activities required for PUFA synthesis is provided together with comparative analyses of bacterial pfa genes and gene products.

Keywords: eicosapentaenoic acid, $p f a$ genes, hydrostatic pressure, polyketide synthase, ribonuclease protection assay

\section{INTRODUCTION}

Omega-3 polyunsaturated fatty acids (PUFAs) such as eicosapentaenoic acid (20:5n-3: EPA) and docosahexaenoic acid (22:6n-3; DHA) are essential components of many animal membrane lipids, well documented for their beneficial effects in human health and

Abbreviations: ACP, acyl carrier protein; AT, acyl CoA:ACP transacylase; $\mathrm{CLF}$, chain length factor; $\mathrm{DHA}$, docosahexaenoic acid; $\mathrm{DH} / \mathrm{I}$, dehydratase/ isomerase; EPA, eicosapentaenoic acid; ER, enoyl reductase; FAS, fatty acid synthase; KR, $\beta$-ketoacyl-ACP reductase; KS, $\beta$-ketoacyl-ACP synthase; PKS, polyketide synthase; PPTase, phosphopantetheinyl transferase; PUFA, polyunsaturated fatty acid; RPA, ribonuclease protection assay.

The GenBank accession numbers for the sequences reported in this paper are AF409100 and AF467805. role as precursors for many hormone and hormonelike regulatory molecules (Angerer \& Schacky, 2000; Lauritzen et al., 2001; Sauer et al., 2001). PUFAs can be obtained directly from dietary sources or synthesized by chain elongation and aerobic desaturation of preexisting fatty acids such as linoleic acid $(18: 2 n-6)$ and $\alpha$ linolenic acid (18:3n-3) (Beaudoin et al., 2000; ParkerBarnes et al., 2000). Marine algae and numerous fungal micro-organisms produce significant quantities of PUFAs via aerobic pathways involving elongation/desaturation (Bajpai \& Bajpai, 1993).

PUFAs were once thought to be absent in bacterial membranes (Erwin \& Bloch, 1964), but numerous bacterial species of marine origin have now been shown to produce very-long-chain PUFAs such as EPA and 
DHA. Such isolates have been found to be particularly prevalent in high-pressure, low-temperature deep-sea habitats and permanently cold marine environments (DeLong \& Yayanos, 1986; Nichols et al., 1993; Yano et al., 1997). The enrichment of PUFA-producing strains from these environments has led to speculation that PUFA synthesis is an important adaptation for countering the effects of elevated hydrostatic pressure and low temperature on membrane fluidity or phase. In strains which have been analysed, PUFA synthesis undergoes temperature-dependent and, for deep-sea isolates, pressure-dependent regulation. Typically, as cultivation temperature is decreased, and/or pressure increased, PUFA incorporation into membrane phospholipids is enhanced. This modulation is thought to maintain appropriate membrane physical structure (Russell \& Nichols, 1999). However, for at least one psychrotolerant piezophilic (high-pressure-adapted) deep-sea bacterium, Photobacterium profundum strain SS9, growth at high pressure and low temperature does not depend upon PUFA synthesis (Allen et al., 1999).

A variety of bacterial fatty acid biosynthetic mechanisms exist which vary with taxonomic identity and class of fatty acid product (Cronan \& Rock, 1996; Fujii \& Fulco, 1977; Rawlings, 1998). Some reports have suggested bacterial omega-3 PUFA production to be mediated by undefined desaturases (Russell \& Nichols, 1999; Tanaka et al., 1999; Watanabe et al., 1997). However, sequence studies of bacterial genes required for PUFA biosynthesis have gradually led to a reappraisal of this view. Initial insight into the genetics of bacterial PUFA synthesis was gained by the cloning and analysis of a $38 \mathrm{kbp}$ genomic fragment from the EPA producer Shewanella sp. strain SCRC-2738 (Yazawa, 1996). Five Shewanella genes, designated ORFs 2, 5, 6, 7 and 8, were shown to be necessary for recombinant EPA synthesis in Escherichia coli and in the marine cyanobacterium Synechococcus sp. (Takeyama et al., 1997; Yazawa, 1996). A subsequent analysis of the predicted amino acid sequences of the products of these genes indicated that they are most related to microbial polyketide synthase (PKS) complexes and fatty acid synthase (FAS) enzymes (Metz et al., 2001). PKS enzymes catalyse the synthesis of a wide array of complex natural products by the repetitive condensation and processing of simple monomeric substrates in a process resembling fatty acid synthesis (Hopwood \& Sherman, 1990). In addition to the Shewanella sp. SCRC-2738 sequences, related genes partially responsible for PUFA production have been analysed from the DHA-producing bacterium Moritella marina strain MP-1 (formerly Vibrio marinus) (Tanaka et al., 1999) and from a DHA-producing thraustochytrid marine protist belonging to the genus Schizochytrium (Metz et al., 2001).

Recently, Metz et al. (2001) reported biochemical analyses of PUFA production in E. coli strains harbouring Shewanella sp. SCRC-2738 DNA and in the Schizochytrium species. Consistent with the examination of enzyme domains, isotopic labelling studies provided compelling support for a PKS-like pathway of PUFA synthesis in both systems studied (Metz et al., 2001). However, whereas considerable advances have been made towards a mechanistic understanding of microbial PUFA production, very little is known about the regulation of PUFA synthesis. The present study reports the cloning and molecular analysis of genes responsible for EPA synthesis, herein referred to as $p f a$ (polyunsaturated fatty acid) genes, from the deep-sea bacterium $P$. profundum strain SS9. Transcriptional regulation of the SS9 $p f a A-D$ genes was analysed as a function of varying temperature and hydrostatic pressure, and SS9 mutants containing polar insertions in two $p f a$ genes were used to verify gene function and to help delineate the transcriptional organization of the $p f a$ operon. Furthermore, an SS9 mutant that overproduces EPA was characterized and found to upregulate $p f a$ gene transcription.

\section{METHODS}

Strains and growth conditions. All strains and plasmids used in this study are listed in Table 1. P. profundum strains were routinely cultured at $15^{\circ} \mathrm{C}, 1$ atmosphere $(1 \mathrm{~atm}=0 \cdot 101 \mathrm{MPa})$ in 2216 Marine Medium (28 g litre; Difco). All temperature experiments $\left(15\right.$ and $\left.4{ }^{\circ} \mathrm{C}\right)$ were conducted aerobically in 2216 Marine Medium. For solid media, agar (Difco) was added at $17 \mathrm{~g} \mathrm{l}^{-1}$. Antibiotics kanamycin $\left(50 \mu \mathrm{g} \mathrm{ml}^{-1}\right.$ for E. coli, $200 \mu \mathrm{g} \mathrm{ml}^{-1}$ for $P$. profundum strains), rifampicin $\left(100 \mu \mathrm{g} \mathrm{ml}^{-1}\right)$ and chloramphenicol $\left(20 \mu \mathrm{g} \mathrm{ml}^{-1}\right)$ were added to media when required. All antibiotics were obtained from Sigma. High-pressure cultivation of $P$. profundum strains for growth studies, fatty acid analyses and extraction of RNA was as previously described (Allen et al., 1999).

Construction of an SS9 genomic fosmid library. Preparation of a genomic library of $P$. profundum strain DB110 in the pFOS1 vector (Kim et al., 1992) was performed as described by Stein et al. (1996). Briefly, high-molecular-mass genomic DNA was isolated from strain DB110 by lysis and extraction in agarose plugs and subsequently digested with Sau3A. DNA fragments between 35 and $45 \mathrm{kbp}$ were purified following gel electrophoresis using the GeneClean Spin Kit (Bio 101). Sizeselected DNA was ligated into the BamHI site of pFOS1 vector arms, in vitro packaged using Gigapack III XL packaging extracts (Stratagene), and transfected to E. coli DH10B. Approximately 960 fosmid clones were individually picked into 96-well microtitre dishes containing LB medium plus $20 \mu \mathrm{g}$ chloramphenicol $\mathrm{ml}^{-1}$ and $10 \%(\mathrm{v} / \mathrm{v})$ glycerol and stored at $-80^{\circ} \mathrm{C}$ until further analysis.

DNA hybridizations and fosmid clone manipulations. Fosmid library clones were replicated onto MagnaCharge (MSI, Westboro, MA, USA) nylon filters and hybridized to DNA probes obtained from internal fragments of SS9 $p f a$ genes using standard protocols (Sambrook et al., 1989). Previously we reported the cloning of an $885 \mathrm{bp}$ internal fragment of the SS9 $p f a A$ gene (designated ORF $3 / 4$ in the previous report) making use of arbitrary primers derived from the Shewanella sp. SCRC-2738 EPA gene sequence (GenBank accession no. U73935) (Allen et al., 1999). An internal fragment of SS9 pfaD was subsequently obtained using primers ORF9-3 (5'-CGTTGAAGCATCAGCTTTCTT-3') and ORF9-2 (5'-TACGCCCATCTCGAACATATC-3') derived from SCRC-2738 EPA gene sequence. The resultant $571 \mathrm{bp}$ PCR product contained a high degree of similarity, $79 \%$ identity at the DNA level, to the SCRC-2738 pfaD homologue (designated ORF7; Yazawa, 
Table 1. Strains and plasmids used in this study

\begin{tabular}{|c|c|c|}
\hline Strain or plasmid & Description & Reference or source \\
\hline \multicolumn{3}{|l|}{$\begin{array}{l}\text { Photobacterium } \\
\text { profundum }\end{array}$} \\
\hline SS9 & $\mathrm{EPA}^{+} ;$wild-type strain & DeLong et al. (1997) \\
\hline DB110 & Lac $^{-}$Rif $^{r}$; SS9 derivative & Chi \& Bartlett (1993) \\
\hline EA2 & EPA-overproducing chemical mutant & Allen et al. (1999) \\
\hline EA10 & $p f a A$ insertion mutant $\left(\mathrm{EPA}^{-}\right)$ & Allen et al. (1999) \\
\hline EA50 & $p f a D$ insertion mutant $\left(\mathrm{EPA}^{-}\right)$ & This study \\
\hline \multicolumn{3}{|l|}{ Shewanella sp. } \\
\hline \multicolumn{3}{|l|}{ Plasmids } \\
\hline pFOS1 & Fosmid cloning vector & Kim et al. (1992) \\
\hline pFOS8E1 & Fosmid clone containing SS9 $p f a A-D$ & This study \\
\hline pMUT100 & Mobilizable suicide plasmid, $\mathrm{Km}^{\mathrm{r}}$ & Brahamsha (1996) \\
\hline pCR2.1 & PCR cloning vector, $\mathrm{Km}^{\mathrm{r}}$ & Invitrogen \\
\hline pDP18 & In vitro transcription vector & Ambion \\
\hline
\end{tabular}

1996)). Filters were initially probed using the internal fragment of SS9 $p f a A$, stripped of bound probe, and reprobed with the $p f a D$ probe. Of the 42 clones to which both probes hybridized, fosmid 8E1 (the clone with the smallest insert size of $33 \cdot 1 \mathrm{kbp}$ ) was selected for further analysis and sequencing. Fosmid DNA was purified using the Qiagen Plasmid Midi kit and digested with NotI to excise the cloned insert. A subclone library of fosmid 8E1 was prepared by digesting the NotI insert with Sau3A, size selecting for 1-2 kbp fragments, and ligation into BamHI-digested pUC18. Plasmid minipreparations of the fosmid 8E1:pUC18 subclones were prepared and sequenced using pUC18-specific primers flanking the cloned inserts.

DNA sequencing and analysis. Double-stranded DNA sequencing reactions were performed using the ABI Prism BigDye Terminator Cycle Sequencing Ready Reaction kit (Applied Biosystems) and run on an Applied Biosystems model 373 DNA sequencing system. Initial sequence analysis and contig assembly was performed using Sequencher 3.1 software (Gene Codes Corp.). Additional sequence needed to fill in contig gaps was obtained using sequence-specific oligonucleotide primers and sequencing of PCR products. Global similarity searches were performed using the BLAST network service (Altschul et al., 1997). Multiple alignments were performed using ClustalW (Higgins \& Sharp, 1988) in conjunction with GeneDoc software (Nicholas \& Nicholas, 1997). Domain arrangement analyses of the predicted amino acid sequences of the $p f a$ genes were conducted using the ProDom database of protein domain families (Corpet et al., 1999), the Conserved Domain Database with Reverse Position Specific BLAST (Altschul et al., 1997) and the ISREC ProfileScan server (http://hits.isb-sib.ch/cgi-bin/hits_motifscan).

Insertional inactivation mutagenesis. Following previously published procedures (Allen \& Bartlett, 2000; Allen et al., 1999), insertional inactivation mutagenesis was performed targeting the SS9 $p f a D$ gene. Briefly, an internal fragment of $p f a D$ was PCR-amplified using primers ORF9-3 and ORF9-2 (sequences listed above; amplified region corresponding to 23708-24278 of GenBank accession no. AF409100), cloned into the pCR2.1-TOPO vector (Invitrogen) and subcloned into the mobilizable suicide vector pMUT100 (Brahamsha,
1996). The $p f a D: p M U T 100$ construct was introduced into SS9 from E. coli by conjugal transfer as described by Chi \& Bartlett (1993). Kanamycin-resistant exconjugants arose from plasmid integration into the SS9 chromosome in a single crossover event yielding strain EA50, with $p f a D$ insertionally inactivated. The site of plasmid insertion was verified by PCR amplification of a portion of the $p f a D$ gene using primers located upstream of the insertion site together with pMUT100specific primers.

Cloning of phosphopantetheinyl transferase (PPTase) genes. The Bacillus subtilis sfp gene (GenBank accession no. X63158) was isolated from B. subtilis by PCR amplification of the complete gene using primers 5'-TGCTGAATTATGCTGTGGCAAGGC-3' and 5'-GCTTCTCGAAATGATGTTCCCCGG-3'. In attempts to isolate PUFA synthase PPTase gene sequences, degenerate PCR primers were designed to conserved PPTase motifs found by alignment of known PPTase protein sequences including the PUFA synthase PPTase of Shewanella sp. strain SCRC-2738 (ORF2; GenBank accession no. U73935). Template DNA isolated from a variety of EPA-producing bacterial strains was employed; however, only DNA from the psychrotolerant, moderate piezophile Shewanella sp. strain SC2A (DeLong et al., 1997) yielded an amplification product of the expected size relative to the SCRC-2738 sequence. Using forward primer 5'-GGCGATAAAGGYAARCCK-3' and reverse primer 5'-CAACGHTCRATRTCWCCACC-3' a 212 bp product was sequenced whose deduced amino acid sequence showed $48 \%$ identity and $61 \%$ similarity over 72 amino acids to the SCRC-2738 ORF2 product. In order to obtain flanking DNA sequence, primers internal to the SC2A PPTase sequence were designed for inverse PCR (Ochman et al., 1990) and an SC2A cosmid library (Chilukuri \& Bartlett, 1997) was screened using the SC2A PPTase internal fragment. Colony hybridizations were performed according to standard protocols (Sambrook et al., 1989). Plasmid DNA was isolated from positively hybridizing clones and sequenced. The complete sequence of the Shewanella sp. strain SC2A PPTase is deposited under GenBank accession no. AF467805. 
Fatty acid analyses. Extraction and analysis of fatty acid methyl ester preparations via combined gas chromatographymass spectroscopy were performed as previously described (Allen et al., 1999). Fatty acids are denoted as number of carbon atoms: number of double bonds.

RNA isolation and ribonuclease protection assay (RPA) analyses. Total RNA was extracted from mid-exponentialphase $P$. profundum strains grown at various temperatures and pressures using the RNAzol B method (Tel-Test, Friendswood, TX, USA). $\left[\alpha_{-}{ }^{32} \mathrm{P}\right] \mathrm{UTP}$-labelled RNA probes were synthesized using the T7 RNA polymerase MAXIscript in vitro transcription kit (Ambion, Austin, TX) and RPAs were performed using the RPA III kit according to the manufacturer's protocols (Ambion). Probe template preparations involved the PCR amplification of fragments of SS9 pfa genes, cloning of PCR products into the pCR2.1-TOPO vector and subsequent subcloning of inserts into the pDP18 transcription vector (Ambion). The sizes of full-length probes and protected fragments were as follows (the positions of $p f a$ RPA probes are indicated with reference to GenBank accession no. AF409100): pfaA 377/268 bp (9422-9689), pfaB 465/322 bp (15332-15653), pfaC 477/344 bp (19133-19476), pfaD 477/ 344 bp (23385-23728), pfaA/B 520/264 bp (15020-15283), pfaB/C 516/260 bp (17129-17388), pfaC/D 511/255 bp (23052-23306). RNA probes were purified from denaturing acrylamide continuous gels, co-precipitated with $10 \mu \mathrm{g}$ total RNA and hybridized overnight at $45^{\circ} \mathrm{C}$. Following RNase treatment, protected fragments were separated on denaturing acrylamide gels $(5 \%$ acrylamide $/ 8 \mathrm{M}$ urea) against undigested probe and appropriate controls. For detection of probes and protected fragments, gels were transferred to filter paper and exposed to X-ray film overnight. $\left[\alpha_{-}{ }^{32} \mathrm{P}\right] \mathrm{UTP}$-labelled RNA Century Markers (Ambion) were used as size standards.

Primer extension analysis. Primer extension analysis of the SS9 $p f a A-D$ genes was performed using the Primer Extension System-AMV Reverse Transcriptase kit (Promega). RNA was isolated from SS9 strain DB110 using the RNAzol B method. Multiple $p f a$ extension primers were tested; however, only $p f a A$ primers 5'-GCCATGCCAACAATCGCAAT-3' (position 7529-7548) and 5'-GTTGCGATTAGGCAACTGGTGA-3' (position 7379-7400) yielded extension products. Primers were end-labelled using $\left[\gamma^{32} \mathrm{P}\right] \mathrm{ATP}$ and T4 polynucleotide kinase. Labelled primers were annealed to $40 \mu \mathrm{g}$ SS9 RNA and extended using AMV-RT. For DNA sequencing, $p f a A$ plasmid templates were constructed using DNA amplified with primers 5'-AACCTCTTGCTCCAGTGATTG-3' and 5'-TATCACGGTCGTATGTTTCCG-3' (amplified fragment position 7067-7856), cloned into pCR2.1, and sequenced using the labelled primers used for primer extension in conjunction with the fmol DNA Cycle Sequencing System Kit (Promega). DNA fragments were resolved on $8 \mathrm{M}$ urea/8\% polyacrylamide gels.

\section{RESULTS}

\section{Isolation and sequence analysis of the $P$. profundum strain SS9 pfa gene cluster}

The isolation of SS9 genes required for EPA synthesis (termed $p f a A-D$; polyunsaturated fatty acid) first involved the generation of a large insert fosmid library of SS9 genomic DNA and the subsequent probing of this library using a partial fragment of the SS9 $p f a A$ gene previously isolated (Allen et al., 1999). Based on omega3 PUFA synthase sequence information from Shewanella sp. SCRC-2738 (GenBank accession no. U73935) and
Moritella marina (GenBank accession no. AB025342), the size of the SS9 gene cluster was predicted to be approximately $18 \mathrm{kbp}$. In order to optimize identification of library clones containing the complete EPA gene cluster from SS9, clones hybridizing positively with the proximal $p f a A$ gene fragment were subsequently probed using an internal fragment of the distal SS9 pfaD gene. Fosmid 8E1 (insert size of $33100 \mathrm{bp}$ ) was found to positively hybridize to both probes and was selected for sequencing. Fig. 1 shows the genetic organization of the 19 predicted ORFs identified within this sequence as well as the size and similarity of these ORFs to other sequences present in GenBank based on BLAST searches. All SS9 sequences have been deposited in GenBank under accession number AF409100.

The SS9 $p f a A-D$ genes span a region of $17347 \mathrm{bp}(p f a A$ 7722 bp, pfaB $2100 \mathrm{bp}$, pfaC 5877 bp, pfaD 1635 bp). The deduced amino acid sequence of the SS9 $p f a$ genes had a high degree of similarity and identity to $p f a$ homologues in Shewanella and Moritella (\% identity/ similarity): SS9 pfaA-62\%/72\% Shewanella, $46 \% /$ $61 \%$ Moritella; SS9 pfaB-44\%/58\% Shewanella, $18 \% / 33 \%$ Moritella; SS9 pfaC-67\%/77\% Shewanella, 45\%/60\% Moritella; SS9 pfaD-82\%/88\% Shewanella, $62 \% / 76 \%$ Moritella. Phylogenetically, SS9 is closely related to both Shewanella and Moritella, and the high degree of similarity of the SS9 and Shewanella $p f a$ gene products likely reflects functional relatedness of the products (i.e. EPA vs DHA). In addition, the predicted proteins possess strong similarity to PKSs from Streptomyces, Mycobacterium and $\mathrm{Ba}$ cillus, as well as cyanobacterial heterocyst glycolipid synthases and eukaryotic FAS enzymes. Comparison of ORFs in SS9, Shewanella and Moritella flanking their respective $p f a$ gene clusters showed no sequence conservation with the exception of SS9 ORF6, which showed a high degree of identity $(85 \%)$ to an ORF also located immediately upstream of $p f a A$ in Moritella (ORF7; GenBank accession no. AB025342). The function of this gene is not known but it possesses homology to a hydroxyacyl-CoA thioesterase (product of the $f c b C$ gene) of Pseudomonas sp. DJ-12 (Chae et al., 2000).

Of the 19 ORFs identified, seven did not possess any significant homology to GenBank sequences and were noted as hypothetical proteins. Upstream of the SS9 $p f a$ genes is a putative methyl-accepting chemotaxis protein (similar to Vibrio cholerae protein VCA093) and a cluster of four genes which appear to be arranged in an operon structure. Included in this cluster is a putative fabH paralogue. We denote this gene as a putative paralogue because a homologue of $f a b H$ has previously been cloned and sequenced from SS9, located within a distinct cluster of genes involved in saturated and monounsaturated fatty acid synthesis, an organization found in numerous $\gamma$-proteobacteria (our unpublished results). In addition, putative haloalkane dehalogenase, acyl-CoA ligase, and steroid dehydrogenase genes were identified within this upstream cluster. Based on BLAST searches, each of these four genes displays the highest similarity to genes in the plant pathogen Xylella 

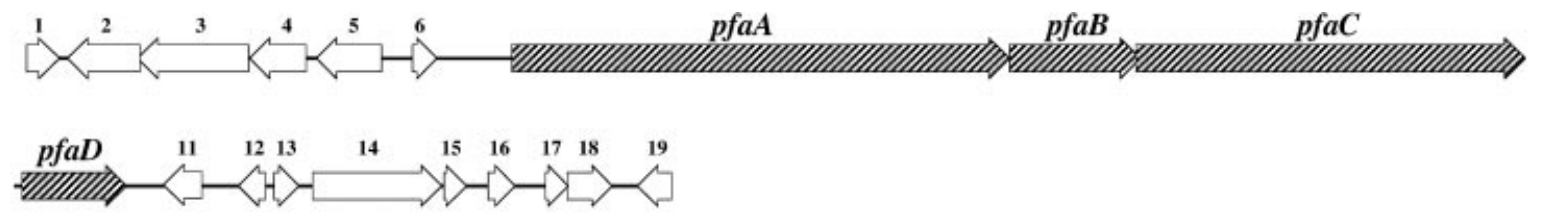

\begin{tabular}{|c|c|c|}
\hline ORF & Size (bp) & Similarity \\
\hline 1 & 513 partial & putative methyl-accepting chemotaxis protein (Vibrio cholerae protein VCA093) \\
\hline 2 & 1116 & putative steroid dehydrogenase (Xylella fastidiosa protein XF1970) \\
\hline 3 & 1755 & putative acyl-CoA ligase (Xylella fastidiosa protein XF2276) \\
\hline 4 & 915 & putative haloalkane dehalogenase (Xylella fastidiosa protein XF1965) \\
\hline 5 & 1071 & putative $f a b H$ paralog; 3-oxoacyl-ACP synthase III (Xylella fastidiosa protein XF1970) \\
\hline 6 & 402 & ORF6; Moritella marina ORF7 homolog; possible hydroxyacyl-CoA thioesterase \\
\hline 7 & 7722 & $p f a A$; Eicosapentaenoic acid $(20: 5 n-3)$ fatty acid synthase $A$ \\
\hline 8 & 2100 & pfaB; Eicosapentaenoic acid (20:5n-3) fatty acid synthase B \\
\hline 9 & 5877 & pfaC; Eicosapentaenoic acid (20:5n-3) fatty acid synthase $\mathrm{C}$ \\
\hline 10 & 1635 & pfaD; Eicosapentaenoic acid (20:5n-3) fatty acid synthase D \\
\hline 11 & 630 & ORF11; hypothetical protein \\
\hline 12 & 414 & ORF12; hypothetical protein \\
\hline 13 & 243 & ORF13; hypothetical protein \\
\hline 14 & 2046 & alkaline serine protease (Vibrio metschnikovii vapT) \\
\hline 15 & 393 & ORF15; hypothetical protein \\
\hline 16 & 477 & ORF16; hypothetical protein \\
\hline 17 & 384 & ORF17; hypothetical protein \\
\hline 18 & 762 & ORF18; hypothetical protein \\
\hline 19 & 556 partial & formyltetrahydrofolate deformylase; purine biosynthesis; (Pseudomonas aeruginosa pur $U$ ) \\
\hline
\end{tabular}

Fig. 1. $P$. profundum strain SS9 pfa gene cluster and flanking DNA. Graphic map showing the organization of the 19 ORFs identified from the sequencing of the $33100 \mathrm{bp}$ genomic insert of fosmid 8E1 together with the size of the identified ORFs and their similarity to sequences present in GenBank based on BLAST searches. All SS9 sequences have been deposited under GenBank accession no. AF409100.

fastidiosa (GenBank accession no. AE003849). Unlike SS9, however, none of these genes are linked in the Xylella genome. Downstream of the SS9 pfa cluster numerous ORFs of unknown function were identified as well as an alkaline serine protease (homologue of Vibrio metschnikovii vapT) and a formyltetrahydrofolate deformylase gene (homologue of Pseudomonas aeruginosa purU) involved in purine biosynthesis.

\section{Analysis of SS9 EPA biosynthetic enzymes}

Similarity searches of the SS9 $p f a$ gene products revealed significant matches to numerous multifunctional enzyme complexes involved in such processes as polyketide antibiotic synthesis (Hopwood \& Sherman, 1990; Pfeifer \& Khosla, 2001), eukaryotic fatty acid synthesis (Beaudoin et al., 2000; Parker-Barnes et al., 2000) and heterocyst glycolipid synthesis (Campbell et al., 1997). Domain analyses within individual $p f a$ gene products also revealed numerous enzyme domains characteristic of functions present in bacterial type II fatty acid synthesis. This type of organization is similar to type I PKSs, multifunctional enzymes containing sets of FASrelated activities for successive rounds of polyketide chain elongation and derivatization (Rawlings, 2001). Fig. 2 shows the domain organization of the SS9, Shewanella and Moritella $p f a A-D$ deduced amino acid sequences. Seven enzyme domains were identified within the $p f a$ products: $\beta$-ketoacyl-ACP synthase (KS), acyl
CoA-ACP transacylase (AT), acyl carrier protein (ACP), $\beta$-ketoacyl-ACP reductase (KR), chain length factor (CLF; possible malonyl-ACP decarboxylase activity Bisang et al., 1999), $\beta$-hydroxyacyl-ACP dehydratase/ isomerase $(\mathrm{DH} / \mathrm{I})$ and enoyl reductase (ER).

The domain organization of the SS9 and Shewanella pfa products was identical with the exception of one additional ACP domain found in Shewanella PfaA (six ACPs compared to five in SS9 PfaA). Pfa domains of the DHA-producing Moritella differed from the EPA-producing SS9 and Shewanella products by the inclusion of one additional KS (or CLF) domain in PfaB. CLF domains are homologous to KS domains but the KS active site has a conserved cysteine residue whereas in CLF a glutamine residue exists (Bisang et al., 1999). In Moritella, PfaB has a region homologous to the Cterminal domain of KS but lacks an active-site sequence. Similarly, the second KS-like domain present in Moritella PfaC lacks both a KS active-site motif and the diagnostic glutamine residue of CLF.

Interestingly, growth of SS9 in the presence of the fungal antibiotic cerulenin, a potent irreversible inhibitor of fatty acid biosynthetic condensing enzymes such as KAS I and KAS II (Omura, 1981), has no effect on EPA production (Allen et al., 1999). This resistance could result from blocked access of cerulenin to the Pfa KS active sites, reflecting structural differences between the Pfa KS domains and type II KAS enzymes. 


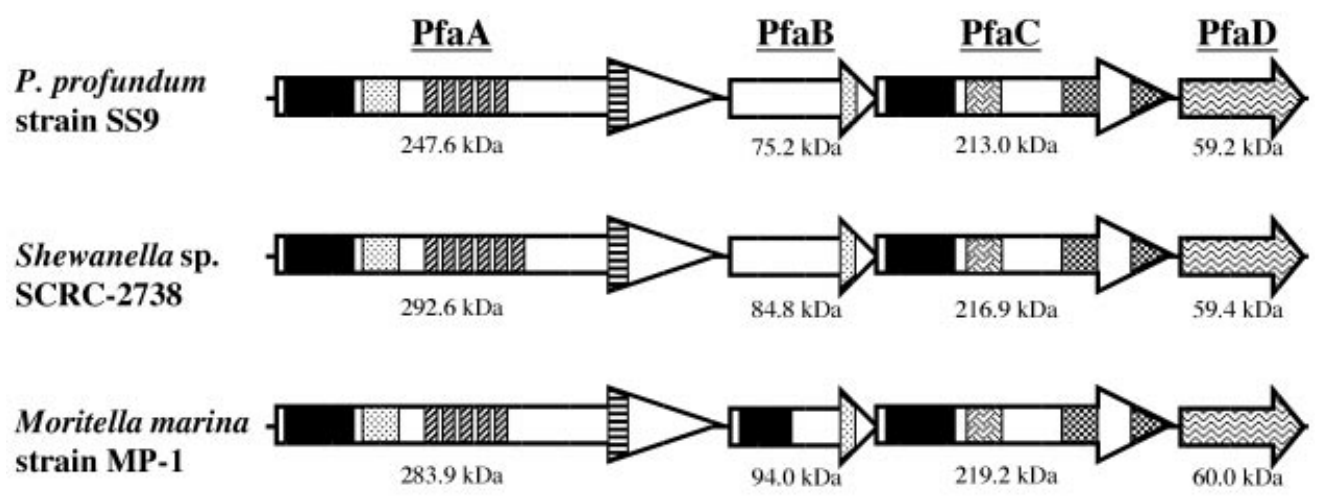

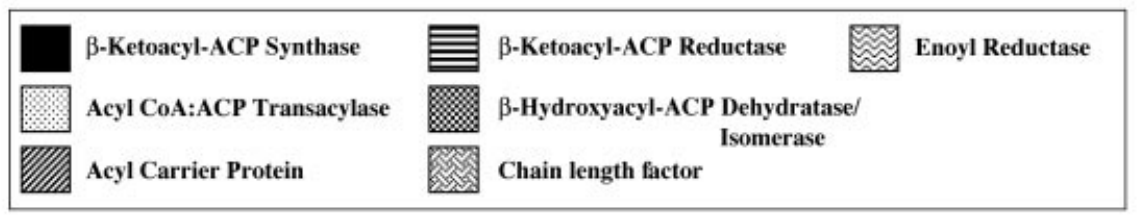

Fig. 2. Comparison of enzyme domains identified within bacterial pfaA-D gene products. $P$. profundum strain SS9 and Shewanella sp. strain SCRC-2738 produce EPA whereas M. marina strain MP-1 produces DHA. Enzyme domains (represented as filled regions) were identified within individual gene products by conserved motif database searches as described in Methods. The predicted sizes of deduced amino acid sequences are noted beneath each product.

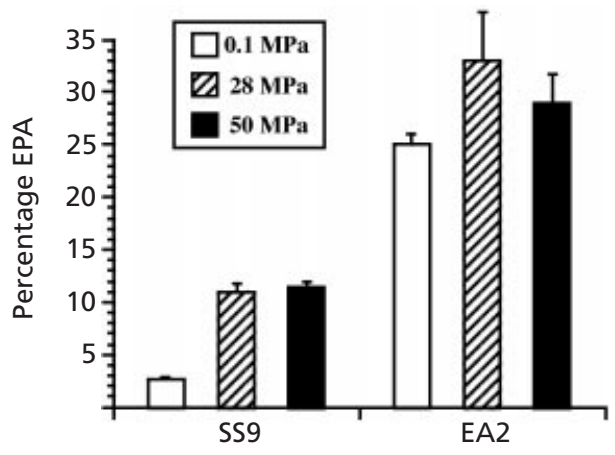

Fig. 3. Effect of varying hydrostatic pressure on EPA percentage composition in wild-type SS9 and chemical mutant strain EA2. Fatty acids were recovered and analysed from mid-exponentialphase cells cultivated at the corresponding pressure $\left(9^{\circ} \mathrm{C}\right)$ as described in Methods. Data represent mean percentage composition (by weight) \pm standard deviation $(n=3)$.

\section{EPA synthesis in SS9 and overproducing strain EA2}

The percentage composition of EPA present in SS9 membranes undergoes temperature- and pressure-dependent modulation (Allen et al., 1999). An increase in cultivation pressure from $0.1 \mathrm{MPa}$ to $28 \mathrm{MPa}$ $[0 \cdot 1 \mathrm{MPa}=1 \mathrm{~atm}=1 \mathrm{bar}]$ results in nearly a fourfold increase in EPA percentage composition (Fig. 3). Similarly, EPA percentage composition undergoes moderate increase in response to reduced cultivation temperature, i.e. $15^{\circ} \mathrm{C}$ to $4{ }^{\circ} \mathrm{C}$ (Allen et al., 1999). Fig. 3 shows the percentage composition of EPA as a function of varying hydrostatic pressure in wild-type SS9 and an SS9 mutant strain found to overproduce EPA. This strain, desig- nated EA2, was isolated as an oleic acid (18:1n-9)requiring auxotrophic chemical mutant (Allen et al., 1999). Strain EA2 constitutively produces EPA at a level nearly fivefold that of wild-type SS9 grown at atmospheric pressure (Fig. 3).

\section{Transcripitonal analyses of the SS9 pfa operon: ribonuclease protection assays and primer extension}

RPAs were performed on SS9 $p f a$ genes with RNA isolated from strains cultivated at various hydrostatic pressures and temperatures (Fig. 4). RPA analyses were chosen due to the potential large size of transcripts and indication of relatively weak expression of the $p f a$ genes from prior Northern blot analysis attempts. No differences in the relative transcript abundance of any of the four $p f a$ genes were detected as a function of varying temperature $\left(15^{\circ} \mathrm{C}\right.$ vs $4{ }^{\circ} \mathrm{C}$, Fig. $4 \mathrm{~B}$, lanes 3 vs 4$)$. RNA extracted from cells cultivated at varying hydrostatic pressure $(0 \cdot 1 \mathrm{MPa}$ vs $28 \mathrm{MPa})$ revealed different results. When cells were grown at elevated pressure, transcript abundance of all $p f a$ genes was considerably less than when cells were grown at atmospheric pressure (Fig. 4B, lanes 5 vs 6), a confounding result given the observed increase in EPA percentage composition as a function of increased hydrostatic pressure (Fig. 3). RPA analyses of $p f a$ gene expression were also performed for the EPAoverproducing strain EA2 (Fig. 4B, lanes 7). Results for EA2 revealed dramatic upregulation of $p f a$ gene transcription compared to wild-type SS9 cultivated under identical conditions $\left(15^{\circ} \mathrm{C}, 0 \cdot 1 \mathrm{MPa}\right)$.

In order to delineate the transcriptional organization of the SS9 $p f a$ gene cluster, RPA analyses were performed on RNA extracted from SS9 grown at $15^{\circ} \mathrm{C}, 0 \cdot 1 \mathrm{MPa}$, 
A)

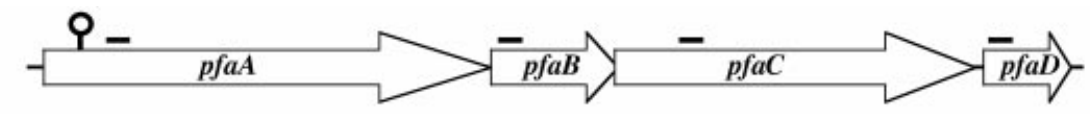

B)

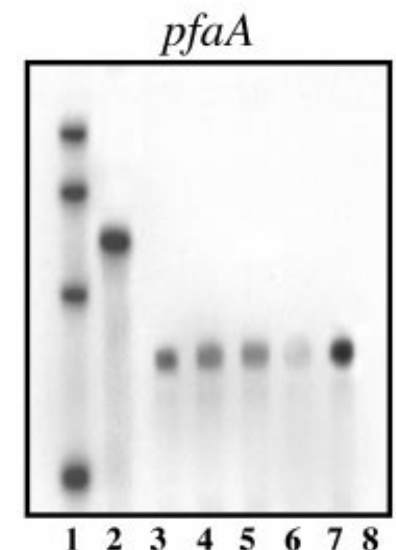

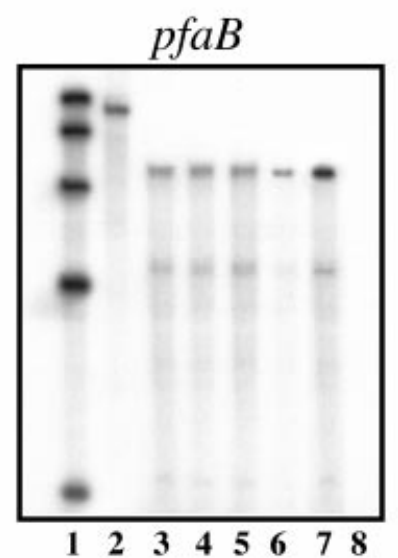

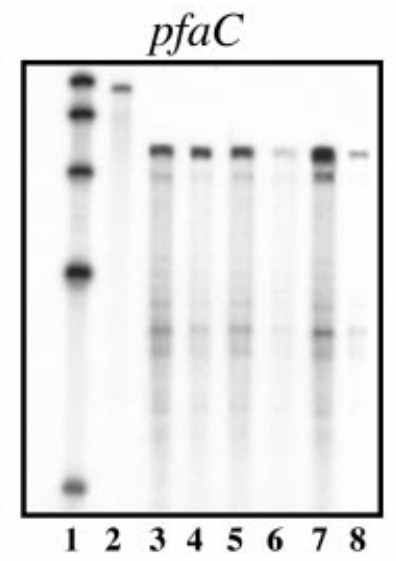

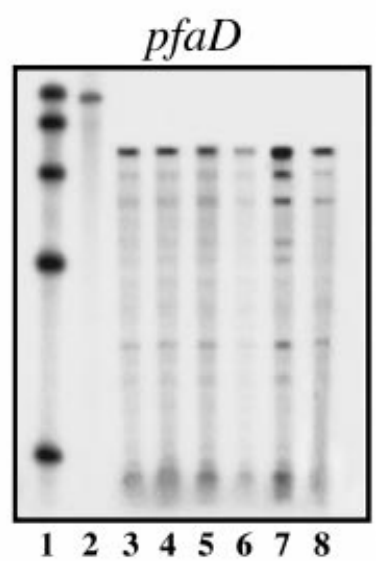

Fig. 4. RPA analyses of SS9 pfaA-D gene transcription. (A) Schematic representation of pfa operon showing location of RPA probes (lines) and site of $p f a A$ insertion in EPA defective mutant strain EA10 (lollipop). (B) RPA results of pfa $A-D$ expression. RPA analyses were performed as described in Methods with $10 \mu \mathrm{g}$ total RNA using ${ }^{32}$ P-labelled riboprobes. Lanes: 1, RNA Century size markers (500, 400, 300, 200, 100 bases); 2, unprotected full-length probes; 3, SS9 at $15{ }^{\circ} \mathrm{C} ; 4$, SS9 at $4{ }^{\circ} \mathrm{C}$; 5 , SS9 at $0.1 \mathrm{MPa}\left(9^{\circ} \mathrm{C}\right) ; 6$, SS9 at $28 \mathrm{MPa}\left(9^{\circ} \mathrm{C}\right) ; 7$, EA2 (EPA-overproducing chemical mutant) at $15^{\circ} \mathrm{C}$; 8 , EA10 (pfaA insertion strain) at $15^{\circ} \mathrm{C}$.

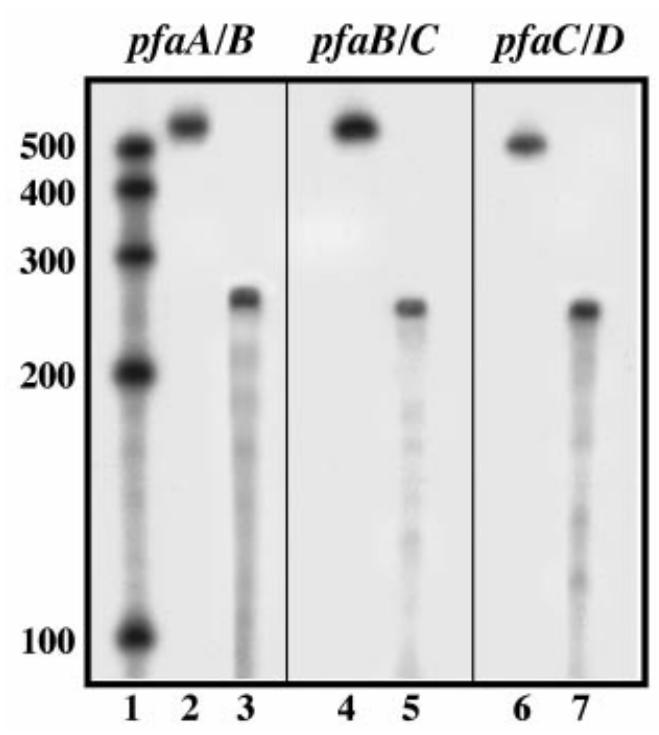

Fig. 5. RPA analysis of SS9 pfa gene linkage: $p f a A / B, p f a B / C$, $p f a C / D$. RPA probes were designed spanning the intergenic regions of adjacent pfa genes. RNA was extracted from SS9 cultivated at $15^{\circ} \mathrm{C}$. Lanes: 1 , RNA Century size maker; 2, 4 and 6 , unprotected full-length probes; 3,5 and 7 , protected fragments.

using probes spanning the putative intergenic regions of the $p f a$ genes (i.e. $p f a A / B, p f a B / C, p f a C / D$; Fig. 5). Linkage RPAs showed protected fragments of the expected size, indicating that adjacent $p f a$ genes are co- transcribed or that promoters for downstream genes are present upstream of the probe-binding sites. Furthermore, transcript analyses were performed using RNA extracted from the $p f a A$ insertion strain EA10. This strain contains a pMUT100 (Brahamsha, 1996) insertion in the $5^{\prime}$ end of $p f a A$. EA10 RPA analyses were used to determine whether there was transcriptional linkage between $p f a A$ and any of the downstream $p f a$ genes. Using RNA from EA10 no RNA protection was detected with the $p f a A$ or $p f a B$ probes. These results indicate that transcription upstream of $p f a A$ drives $p f a A$ and $p f a B$ cotranscription. Analysis of $p f a C$ expression in this strain showed a faint protected fragment suggesting the presence of a weak promoter located in the region spanning the sites of the $p f a B$ and $p f a C$ probes. Finally, a $p f a D$ product of comparable intensity was present using RNA from wild-type SS9 or EA10, indicating the likelihood of a promoter between the region of the $p f a C$ and $p f a D$ probes. RPA analyses thus revealed two major promoter regions and one minor promoter region driving expression of the SS9 $p f a$ gene cluster, giving rise to $p f a A-C, p f a C$ and $p f a D$ transcripts.

In order to localize these promoter regions, primer extension reactions were performed at multiple sites within each of the $p f a$ genes. All attempts to determine transcriptional start sites within $p f a B, p f a C$ and $p f a D$ failed to reveal extension products even with RNA concentrations up to $100 \mu \mathrm{g}$ total RNA. The lack of detection of $p f a C$ and $p f a D$ transcription initiation sites, despite the evidence for $p f a C$ and $p f a D$ promoters, presumably stems from low transcript abundance or stability. However, the transcriptional start upstream of 


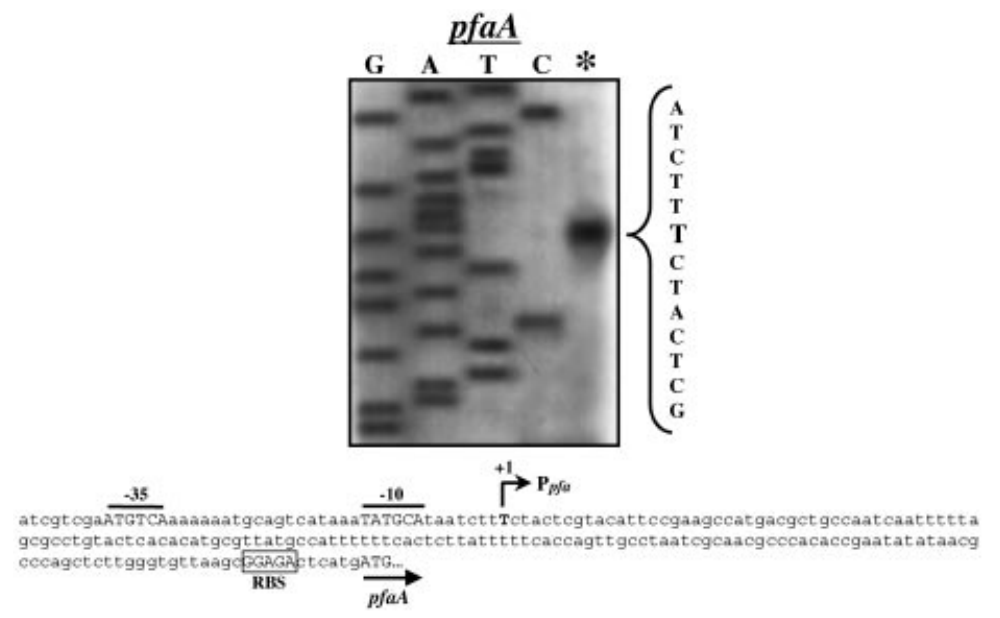

Fig. 6. Determination of SS9 $p f a A$ transcriptional start site by primer extension analysis. The $p f a A$ extension product (lane denoted by an asterisk) was detected using the ${ }^{32} \mathrm{P}$-labelled primer located at position -75 to -54 relative to the translational start of $p f a A$. Dideoxy DNA sequencing reaction mixtures using the same primer were electrophoresed in parallel (lanes G, A, $T$ and $\mathrm{C}$ ). The nucleotide sequence of the transcribed strand immediately flanking the transcriptional start $\mathrm{T}$ is bracketed to the right of the sequencing ladder. The nucleotide sequence and promoter features of the upstream $p f a A$ region are shown below the primer extension analysis results.

$p f a A$ was readily mapped (Fig. 6). $p f a A$ primer extensions were performed with primers corresponding to positions +75 to +94 and -75 to -54 with respect to the $5^{\prime}$ end of $p f a A$. Both primers provided identical results indicating transcription initiation $167 \mathrm{bp}$ upstream of the $p f a A$ ATG translational start (Fig. 6 shows results obtained with the -75 to -54 primer). Putative -35 (ATGTCA) and - 10 (TATGCA) regions were identified as well as the putative ribosome-binding site (GGAGA) located at position -11 to -7 .

\section{Insertional inactivation mutagenesis of the SS9 pfaD gene}

In an analogous manner to the isolation of $p f a A$ insertion mutant EA10, a mutant containing an insertionally inactivated $p f a D$ was constructed, resulting in strain EA50. This $p f a D$ mutant, like the $p f a A$ insertion strain, did not produce EPA under any culture conditions, nor did it accumulate any fatty acids that could serve as intermediates in the EPA biosynthetic pathway (data not shown). Thus, both $p f a$ operons are required for EPA biosynthesis. Mutant EA50 also shared with EA10 the phenotype of no growth defect when cultivated at elevated hydrostatic pressures or reduced temperatures despite its gross alteration in fatty acid composition.

\section{Introduction of PPTase genes into $E$. coli harbouring SS9 pfaA-D}

In an attempt to achieve recombinant EPA production in E. coli we sought to obtain a gene encoding the last remaining enzyme activity needed: PPTase activity. PPTase genes were recovered from both the EPAproducing deep-sea Shewanella sp. strain SC2A (DeLong et al., 1997) and B. subtilis. Based on BLAst analyses, the SC2A putative PPTase shares $55 \%$ identity $(89 / 161$ amino acids) with the Shewanella sp. strain SCRC-2738 PUFA synthase PPTase (ORF2) shown to be required for recombinant EPA production in E. coli host strains (Metz et al., 2001). Introduction of either the SC2A PPTase gene or the $s f p$ gene, the surfactin-synthetase- activating PPTase of B. subtilis (Nakano et al., 1992), into E. coli strains expressing SS9 $p f a A-D$ from pFOS8E1 consistently failed to yield recombinant EPA synthesis.

\section{DISCUSSION}

Progress towards understanding microbial PUFA production has benefited from the recent identification and characterization of genes involved in their synthesis (Metz et al., 2001; Tanaka et al., 1999; Yazawa, 1996). These analyses have provided clues to the mode of synthesis, although biochemical investigations will be needed to resolve many aspects of the pathway. The organization of microbial PUFA synthases is in stark contrast to known eukaryotic PUFA synthesis mechanisms involving elongases and position-specific, oxygen-dependent desaturases (Napier \& Michaelson, 2001; Parker-Barnes et al., 2000). Furthermore, numerous PUFA-producing bacterial strains, including SS9, are capable of producing PUFAs under strictly anaerobic conditions, thus precluding the involvement of an oxygen-dependent mechanism (our unpublished results; Metz et al., 2001; Nichols et al., 1992). Based on the identification of multiple enzyme domains within $p f a$ gene products and ${ }^{13} \mathrm{C}$-labelling studies (Metz et al., 2001) it is now evident that PUFA production shares many features with polyketide synthesis. Analysis of the four SS9 $p f a$ gene products has revealed 10 regions possessing enzyme activities akin to those found in PKS and FAS systems (Fig. 2). The activities of these integrated domains include condensation reactions (KS domains), acyl CoA:ACP transfer reactions (AT), multiple acyl carrier protein domains (ACP), ketoacyl reduction reactions (KR), chain length factor domains (CL) presumably involved in decarboxylation reactions, dehydratase/isomerase reactions $(\mathrm{DH} / \mathrm{I})$, and enoyl reduction reactions (ER). These domains presumably catalyse the reiterative steps in the processing of the growing acyl chain, where each condensation reaction is followed by complete or abbreviated reductive reaction cycles of ketoreduction, dehydration/isomerization, and enoyl reduction. 
The ability to introduce multiple double bonds into a single acyl chain in the absence of desaturation reactions likely arises from the activities of the $\mathrm{DH} / \mathrm{I}$ domains present in the microbial PUFA synthases (bacterial PfaC homologues and Schizochytrium ORF C). Such dehydration/isomerization reactions would be analogous to those catalysed by FabA ( $\beta$-hydroxydecanoyl-ACP dehydratase) in bacterial monounsaturated fatty acid synthesis (Cronan \& Rock, 1996). By dehydration of the $\beta$ hydroxyacyl-ACP substrate, product of $\beta$-ketoacyl-ACP synthase condensation and subsequent reduction of the $\beta$-ketoester by $\beta$-ketoacyl-ACP reductase, a trans double bond is introduced into the growing acyl chain. In selective rounds of acyl chain elongation these double bonds are either preserved by isomerization to the cis form to form an unsaturated acyl-ACP or reduced by an enoyl reductase to a saturated acyl-ACP. Metz et al. (2001) propose a hypothetical pathway for EPA synthesis in Shewanella sp. SCRC-2738 wherein positionspecific isomerases (trans-2,cis-3 and trans-2,cis-2) are involved in trans/cis double bond isomerization. Such a mechanism may be consistent with two DH/I domains being present in PfaC homologues (Fig. 2). Alternatively, the two DH/I domains could be analogous to the FabA and FabZ $\beta$-hydroxyacyl-ACP dehydratase isozymes found in E. coli which differ in reactivity and specificity (Heath \& Rock, 1996).

Unique to microbial PUFA synthases is the presence of clustered repetitive ACP domains (Fig. 2): SS9 $p f a A$ possesses five ACP domains, Shewanella sp. has six, Moritella has five, and Schizochytrium has nine (Metz et al., 2001; Tanaka et al., 1999). Intermediates in the biosynthetic process are presumably bound to these ACP domains as thioesters with AT domains being required for the loading of the starter and extender units. The significance of the disparity in the number of ACP repeats among the PUFA synthase systems is unknown.

Currently, we have been unable to achieve recombinant EPA synthesis in E. coli with the introduced SS9 $p f a A-D$ genes. This shortcoming arises from the need for an additional gene whose product is required for the posttranslational modification of the constituent ACP domains present in PfaA. This activity is achieved by a PPTase which converts apo-ACP to its active holo-form by transfer of a $4^{\prime}$-phosphopantetheinyl moiety from coenzyme A to ACP (Lambalot et al., 1996). In Shewanella sp. SCRC-2738 a fifth gene, designated ORF2, required for recombinant EPA synthesis in E. coli has been identified as a PPTase and is located within close proximity of the $p f a A-D$ operons (Metz et al., 2001). Unlike SCRC-2738, the PUFA synthase PPTase is unlinked from the other $p f a$ genes in SS9 and Moritella. We have been unable to clone the ORF2 homologue from either SS9 or Moritella. During attempts to obtain recombinant EPA synthesis, we introduced two PPTase genes into E. coli harbouring SS9 $p f a A-D, B$. subtilis sfp and a Shewanella ORF2 homologue. The B. subtilis sfp gene, involved in surfactin biosynthesis, encodes a PPTase with a broad substrate recognition spectrum
(Nakano et al., 1992; Reuter et al., 1999). In addition, a PPTase homologue which contained a high degree of identity to Shewanella sp. SCRC-2738 ORF2 was cloned and sequenced from the EPA producer Shewanella sp. strain SC2A. Expression of either of these genes in E. coli harbouring SS9 $p f a A-D$ failed to yield recombinant EPA synthesis, suggesting a high degree of specificity of individual PPTases to their cognate ACPs.

While substantial progress has been made towards a mechanistic understanding of microbial PUFA synthesis, very little information exists regarding the regulation of bacterial PUFA production. In those organisms that have been studied, modulation of PUFA percentage composition occurs during changes in cultivation temperature or pressure. For example, growth of SS9 at a hydrostatic pressure of $28 \mathrm{MPa}$ results in an approximately fourfold increase in EPA percentage composition relative to growth at $0 \cdot 1 \mathrm{MPa}$ (Fig. 3). At the outset of our studies, one possibility was that this modulation was the result of transcriptional regulation of the EPA biosynthetic genes.

RPA analyses performed on each of the SS9 $p f a$ genes using RNA extracted from SS9 cells cultivated at various temperatures and pressures revealed that the $p f a$ genes are not transcriptionally regulated in an adaptive manner in response to these parameters (Fig. 4). The observed reduction in $p f a$ transcript abundance at elevated pressure is confounding and could result from diminished transcription initiation or increased transcript turnover at high pressure. Numerous prokaryotic species regulate percentage composition of particular membrane fatty acids in response to cultivation parameters. In E. coli increased cis-vaccenic acid (18:1n-11) composition at low temperature is an intrinsic property of the fatty acid biosynthetic enzyme KAS II ( $\beta$-ketoacylACP synthase II), product of the fabF gene, and a similar regulatory mechanism may account for increased cisvaccenic acid composition at high pressure in SS9 (Allen \& Bartlett, 2000; Cronan \& Rock, 1996). In both bacteria, $f a b F$ is not transcriptionally regulated and, at least for the E. coli enzyme, it is the relative activity of the enzyme at different temperatures that is responsible for the increased production of $18: 1$ at low temperature. Hence, the possibility exists that PUFA synthases exhibit temperature/pressure-responsive characteristics.

Transcriptional analyses indicate that the $p f a$ gene cluster is organized into two operons, $p f a A-C$ and $p f a D$ (Figs 4 and 5). Evidence in support of this conclusion includes the presence of overlapping start/stop codons of adjacent genes, RPA results with probes spanning intergenic regions, and transcript analyses of a strain containing a polar insertion within $p f a A$. The transcriptional start of $p f a A$ has been mapped to 169 bp upstream of the translational start (Fig. 6).

Results from SS9 suggest that the pathway for PUFA synthesis is separate and distinct from the type II FAS producing monounsaturated and saturated fatty acids (Allen \& Bartlett, 2000; Allen et al., 1999). Many of the type II FAS genes have been cloned and sequenced from 
SS9 and Moritella (Allen \& Bartlett, 2000; Tanaka et al., 1999). Metz et al. (2001) reported a probable PUFA synthetic mechanism reliant on malonyl-CoA derived from acetate as would be expected for the type II FAS system. An interesting question is the 'cross-talk' that exists between the two systems with regard to coordinated expression and lipid incorporation. Some initial insight into this interplay has been provided by $p f a$ transcript analysis of an SS9 mutant strain, designated EA2, originally isolated as an oleic acid auxotrophic chemical mutant that overproduces EPA nearly fivefold compared to wild-type SS9 (Fig. 3). In addition, this strain greatly underproduces monounsaturated fatty acids (MUFAs) (Allen et al., 1999). pfa transcript analyses in this strain reveal substantial $p f a A-D$ overexpression relative to wild-type SS9 (Fig. 4). While the nature of the mutation in this strain has yet to be resolved, two opposing hypotheses can be proposed. Either this strain harbours a lesion resulting in decreased MUFA production which results in compensatory increases in $p f a$ transcription and EPA synthesis, or the mutation results in overexpression of both $p f a$ operons and the cellular response is decreased MUFA synthesis. Both models require the presence of a transcription factor that modulates $p f a$ gene expression.

The high degree of sequence similarity between the bacterial (Shewanella sp SCRC-2738, M. marina and SS9) and the eukaryotic microbe Schizochytrium $p f a$ genes suggests the possible involvement of horizontal gene transfer in the acquisition of the $p f a$ gene clusters in the marine environment. However, among the three bacterial strains whose $p f a$ gene clusters have been cloned and sequenced no sequence conservation flanking the $p f a$ clusters is observed with the exception of a single undefined ORF located upstream of $p f a A$ in SS9 and Moritella. Furthermore, there is no apparent GC bias among the $p f a A-D$ genes nor is there indication of flanking genes possessing functions which could facilitate horizontal transfer.

Located upstream of the SS9 $p f a$ cluster resides an intriguing cluster of four genes which appear to be organized into a possible operon (Fig. 1). Included in this cluster is a putative fabH (3-oxoacyl-ACP synthase III; KAS III) paralogue. This $f a b H$ paralogue is distinct from the $f a b$ cluster $f a b H$ homologue, involved in type II fatty acid biosynthesis initiation, which we have cloned and sequenced from SS9 (our unpublished results). Multiple $\mathrm{fabH}$-like sequences have been identified in a few bacterial species including B. subtilis (yjaX, GenBank accession no. F69842, and $y b f B, \mathrm{Y} 14083)$ and $V$. cholerae (GenBank accession nos A82423 and H82128). Within this cluster also reside a putative haloalkane dehalogenase, a probable acyl-CoA ligase, and a putative steroid dehydrogenase/isomerase. Proteins of the hydroxysteroid dehydrogenase/isomerase family (Labrie et al., 1992) are unusual in prokaryotic organisms, with only a single other bacterial homologue having been identified in the plant pathogen Xylella fastidiosa (GenBank accesssion no. AE004004). The sequence and possible operon structure of this gene cluster suggest that their products could function in a common metabolic process including some aspect of fatty acid physiology. Curiously, upstream of the $M$. marina $p f a$ gene cluster lie two genes presumably involved in fatty acid metabolism as well, a 3-ketoacylCoA thiolase/acetyl-CoA acetyltransferase and a probable lipid A acyltransferase (ORFs 1 and 3, GenBank accession no. AB025342).

\section{ACKNOWLEDGEMENTS}

We thank Dr Jefferey L. Stein for providing us with the pFOS1 vector and Alison Yen-Ling Hsieh for assistance in DNA sequencing. We are grateful to the National Science Foundation Metabolic Biochemistry Program (NSF 9974528) for support of this research.

\section{REFERENCES}

Allen, E. E. \& Bartlett, D. H. (2000). FabF is required for piezoregulation of $c i s$-vaccenic acid levels and piezophilic growth in the deep-sea bacterium Photobacterium profundum strain SS9. J Bacteriol 182, 1264-1271.

Allen, E. E., Facciotti, D. \& Bartlett, D. H. (1999). Monounsaturated but not polyunsaturated fatty acids are required for growth of the deep-sea bacterium Photobacterium profundum SS9 at low temperature and high pressure. Appl Environ Microbiol 65, 1710-1720.

Altschul, S. F., Madden, T. L., Schaffer, A. A., Zhang, J., Zhang, Z., Miller, W. \& Lipman, D. J. (1997). Gapped BLAST and PSI-BLAST: a new generation of protein database search programs. Nucleic Acids Res 25, 3389-3402.

Angerer, P. \& Schacky, C. V. (2000). n-3 polyunsaturated fatty acids and the cardiovascular system. Curr Opin Clin Nutr Metab Care 3, 439-445.

Bajpai, P. \& Bajpai, P. K. (1993). Eicosapentaenoic acid (EPA) production from microorganisms: a review. J Biotechnol 30, 161-183.

Beaudoin, R., Michaelson, L. V., Hey, S. J., Lewis, M. J., Shewry, P. R., Sayanova, O. \& Napier, J. A. (2000). Heterologous reconstitution in yeast of the polyunsaturated fatty acid biosynthetic pathway. Proc Natl Acad Sci US A 97, 6421-6426.

Bisang, C., Long, P. F., Cortes, J. \& 7 other authors (1999). A chain initiation factor common to both modular and aromatic polyketide synthases. Nature 401, 502-505.

Brahamsha, B. (1996). A genetic manipulation system for oceanic cyanobacteria of the genus Synechococcus. Appl Environ Microbiol 62, 1747-1751.

Campbell, E. L., Cohen, M. F. \& Meeks, J. C. (1997). A polyketidesynthase-like gene is involved in the synthesis of heterocyst glycolipids in Nostoc punctiforme strain ATCC 29133. Arch Microbiol 167, 251-258.

Chae, J. C., Kim, Y., Kim, Y. C., Zylstra, G. J. \& Kim, C. K. (2000). Genetic structure and functional implication of the $f c b$ gene cluster for hydrolytic dechlorination of 4-chlorobenzoate from Pseudomonas sp. DJ-12. Gene 258, 109-116.

Chi, E. \& Bartlett, D. H. (1993). Use of a reporter gene to follow high-pressure signal transduction in the deep-sea bacterium Photobacterium sp. strain SS9. J Bacteriol 175, 7533-7540.

Chilukuri, L. N. \& Bartlett, D. H. (1997). Isolation and characterization of the gene encoding single-stranded-DNA-binding protein (SSB) from four marine Shewanella strains that differ in 
their temperature and pressure optima for growth. Microbiology 143, 1163-1174.

Corpet, F., Gouzy, J. \& Kahn, D. (1999). Recent improvements of the ProDom database of protein domain families. Nucleic Acids Res 27, 263-267.

Cronan, J. E. \& Rock, C. O. (1996). Biosynthesis of membrane lipids. In Escherichia coli and Salmonella typhimurium: Cellular and Molecular Biology, pp. 612-636. Edited by F. C. Neidhardt and others. Washington, DC: American Society for Microbiology.

DeLong, E. F. \& Yayanos, A. A. (1986). Biochemical function and ecological significance of novel bacterial lipids in deep-sea procaryotes. Appl Environ Microbiol 51, 730-737.

DeLong, E. F., Franks, D. G. \& Yayanos, A. A. (1997). Evolutionary relationships of cultivated psychrophilic and barophilic deep-sea bacteria. Appl Environ Microbiol 63, 2105-2108.

Erwin, J. \& Bloch, K. (1964). Biosynthesis of unsaturated fatty acids in microorganisms. Science 143, 1006-1012.

Fujii, D. K. \& Fulco, A. J. (1977). Biosynthesis of unsaturated fatty acids in Bacilli: hyperinduction and modulation of desaturase synthesis. J Biol Chem 252, 3660-3670.

Heath, R. J. \& Rock, C. O. (1996). Roles of the FabA and FabZ $\beta$ hydroxyacyl-acyl carrier protein dehydratases in Escherichia coli fatty acid biosynthesis. J Biol Chem 271, 27795-27801.

Higgins, D. G. \& Sharp, P. M. (1988). ClustaL: a package for performing multiple sequence alignment on a microcomputer. Gene 73, 237-244.

Hopwood, D. A. \& Sherman, D. H. (1990). Molecular genetics of polyketides and its comparison to fatty acid biosynthesis. Annu Rev Genet 24, 37-66.

Kim, U.-J., Shizuya, H., de Jong, P. J., Birren, B. \& Simon, M. (1992). Stable propagation of cosmid sized human DNA inserts in an F factor based vector. Nucleic Acids Res 20, 1083-1085.

Labrie, F., Simard, J., Luuthe, V. \& 13 other authors (1992). Structure and tissue-specific expression of 3 beta-hydroxysteroid dehydrogenase/5-ene-4-ene isomerase genes in human and rat classical and peripheral steroidogenic tissues. J Steroid Biochem Mol Biol 41, 421-438.

Lambalot, R. H., Gehring, A. M., Flugel, R. S. \& 6 other authors (1996). A new enzyme superfamily - the phosphopantetheinyl transferases. Chem Biol 3, 923-936.

Lauritzen, L., Hansen, H. S., Jorgensen, M. H. \& Michaelsen, K. F. (2001). The essentiality of long chain $n$-3 fatty acids in relation to development and function of the brain and retina. Prog Lipid Res 40, 1-94.

Metz, J. G., Roessler, P., Facciotti, D. \& 10 other authors (2001). Production of polyunsaturated fatty acids by polyketide synthases in both prokaryotes and eukaryotes. Science 293, 290-293.

Nakano, M. M., Corbell, N., Besson, J. \& Zuber, P. (1992). Isolation and characterization of $s f p$ : a gene that functions in the production of the lipopeptide biosurfactant, surfactin, in Bacillus subtilis. Mol Gen Genet 232, 313-321.

Napier, J. A. \& Michaelson, L. V. (2001). Towards the production of pharmaceutical fatty acids in transgenic plants. J Sci Food Agric 81, 883-888.

Nicholas, K. B. \& Nicholas, H. B. J. (1997). GeneDoc. Pittsburg, PA: Pittsburg Supercomputing Center.
Nichols, D. S., Nichols, P. D. \& McMeekin, T. A. (1992). Anaerobic production of polyunsaturated fatty acids by Shewanella putrefaciens strain ACAM 342. FEMS Microbiol Lett 98, 117-122.

Nichols, D. S., Nichols, P. D. \& McMeekin, T. A. (1993). Polyunsaturated fatty acids in Antarctic bacteria. Antarctic Sci 2, 149-160.

Ochman, H., Medhora, M. M., Garza, D. \& Hartle, D. L. (1990). Amplification of flanking sequences by inverse PCR. In PCR Protocols: a Guide to Methods and Application, pp. 219-227. Edited by M. A. Innis and others. London: Academic Press.

Omura, S. (1981). Cerulenin. Methods Enzymol 72, 520-532.

Parker-Barnes, J. M., Das, T., Bobik, E. \& 5 other authors (2000). Identification and characterization of an enzyme involved in the elongation of $n-6$ and $n-3$ polyunsaturated fatty acids. Proc Natl Acad Sci U S A 97, 8284-8289.

Pfeifer, B. A. \& Khosla, C. (2001). Biosynthesis of polyketides in heterologous hosts. Microbiol Mol Biol Rev 65, 106-118.

Rawlings, B. J. (1998). Biosynthesis of fatty acids and related metabolites. Nat Prod Rep 15, 275-308.

Rawlings, B. J. (2001). Type I polyketide biosynthesis in bacteria (Part B). Nat Prod Rep 18, 231-281.

Reuter, K., Mofid, M. R., Marahiel, M. A. \& Ficner, R. (1999). Crystal structure of the surfactin synthetase-activating enzyme Sfp: a prototype of the $4^{\prime}$-phosphopantetheinyl transferase superfamily. EMBO J 18, 6823-6831.

Russell, N. J. \& Nichols, D. S. (1999). Polyunsaturated fatty acids in marine bacteria - a dogma rewritten. Microbiology 145, 767-779.

Sambrook, J., Fritsch, E. F. \& Maniatis, T. (1989). Molecular Cloning: a Laboratory Manual, 2nd edn. Cold Spring Harbor, NY: Cold Spring Harbor Laboratory.

Sauer, L. A., Dauchy, R. T. \& Blask, D. E. (2001). Polyunsaturated fatty acids, melatonin, and cancer prevention. Biochem Pharmacol 61, 1455-1462.

Stein, J. L., Marsh, T. L., Wu, K. E., Shizuya, H. \& DeLong, E. F. (1996). Characterization of uncultivated prokaryotes: isolation and analysis of a 40-kilobase-pair genome fragment from a planktonic marine archaeon. J Bacteriol 178, 591-599.

Takeyama, H., Takeda, D., Yazawa, K., Yamada, A. \& Matsunaga, T. (1997). Expression of the eicosapentaenoic acid synthesis gene cluster from Shewanella sp. in a transgenic marine cyanobacterium Synechococcus sp. Microbiology 143, 2725-2731.

Tanaka, M., Ueno, A., Kawasaki, K. \& 6 other authors (1999). Isolation of clustered genes that are notably homologous to the eicosapentaenoic acid biosynthesis gene cluster from the docosahexaenoic acid-producing bacterium Vibrio marinus strain MP-1. Biotechnol Lett 21, 939-945.

Watanabe, K., Yazawa, K., Kondo, K. \& Kawaguchi, A. (1997). Fatty acid synthesis of an eicosapentaenoic acid-producing bacterium: de novo synthesis, chain elongation, and desaturation systems. J Biochem 122, 467-473.

Yano, Y., Nakayama, A. \& Yoshida, K. (1997). Distribution of polyunsaturated fatty acids in bacteria present in intestines of deep-sea fish and shallow-sea poikilothermic animals. Appl Environ Microbiol 63, 2572-2577.

Yazawa, K. (1996). Production of eicosapentaenoic acid from marine bacteria. Lipids (Supplement) 31, S297-S300.

Received 29 October 2001; revised 18 January 2002; accepted 4 February 2002. 